\title{
Ferrite and Copper Electroless Plating of Photopolymerized Resin for Micromolding of Three-Dimensional Structures
}

Kohki Mukai, Shinya Kitayama, Toshiya Yoshimura, and Shoji Maruo

Department of Mechanical Engineering and Materials Science, Yokohama National University, 79-5 Tokiwadai, Hodogaya-ku, Yokohama, 240-8501, Japan

*E-mail address: mukai@ynu.ac.jp

The electroless plating of ferrite and copper onto photopolymerized resin was investigated with the aim of fabricating three-dimensional microstructures. Evaluation by X-ray diffraction and X-ray fluorescence measurements clarified that the materials were successfully plated onto the resin mold by controlling the temperature and $\mathrm{pH}$ of the source solution. High-resolution optical microscope observation revealed that the $2-\mu \mathrm{m}$-deep pits at the surface were fully plated by the materials, demonstrating the submicron resolution of the microfabrication process. We performed the transcription of three-dimensional structures made of plated copper from a resin mold, and succeeded in the fabrication of a screw. The $40-\mu \mathrm{m}$-thick thread was formed accurately.

KEYWORDS: electroless plating, photopolymerization, mold, micromachine, ferrite, copper 


\section{Introduction}

With the aim of developing viable micromachining technologies, many microstructure formation methods have been developed. Among them, photon-initiated polymerization is very attractive, because it enables the fabrication of complicated three-dimensional structures at low cost ${ }^{1,2)}$. The resolution of the resin structure can be set at less than $100 \mathrm{~nm}$ using two-photon stereolithography ${ }^{3)}$. However, the polymer is not tolerant to chemical and heat treatment. These problems are partially solved by the metal coating of the polymerized resin ${ }^{4,5)}$. The resulting tolerance is better than that of the original, but it is still not sufficiently high because the internal material is a polymer. To avoid the drawbacks of photopolymers, Tanaka et al. developed a technique of directly forming metal structures based on a photochemical reaction ${ }^{6}$. Photons separate out the metal directly on the substrate in the solvent. However, because the reaction field is difficult to control in the solvent, the structural surface shows a roughness of several microns.

We have proposed a new microstructure fabrication method using metal or metal compounds ${ }^{7,8)}$. The fabrication process is as follows. First, a resin mold is formed by photon-initiated polymerization. Next, the resin mold is plated with a metal or metal compound through electroless plating, because the mold is nonconductive. Then, electrolytic grinding is performed to remove any superfluous metal from the surface and expose the inner structure. Because, in principle, electrolytic grinding cannot progress when the nonconductive resin mold is exposed, there is no excessive removal of metal even in the case of highly detailed features. Finally, the inner metal structure is extracted by removing the resin mold. The ability to form three-dimensional microstructures made of metal or metal compounds will open up various application fields of 
micromachining, owing to the special properties of such microstructures, including mechanical strength, shape memory, magnetism, high conductivity, and surface plasmon state. If we can put various types of material together using this molding technique, multifunctional micromachines will be realized. In our previous studies, we tested the micromolding process using nickel. In this work, we investigated electroless plating using ferrite and copper, and fabricated a small screw through the abovementioned molding process.

\section{Ferrite Electroless Plating}

We studied the electroless plating of ferrite onto photopolymerized resin and the characteristics of the plated film. Pellet-shaped samples were cured with an UV lamp. Before plating, we cleaned the samples in water and acetone using ultrasonic waves. Then, the samples were immersed in a bath, as shown in Figure 1. The $1.5 \mathrm{~g} / \mathrm{L} \mathrm{FeCl}_{2} \cdot 4 \mathrm{H}_{2} \mathrm{O}+$ $1.8 \mathrm{~g} / \mathrm{L} \mathrm{CoCl} 2 \cdot 6 \mathrm{H}_{2} \mathrm{O}$ plating solution and the $0.35 \mathrm{~g} / \mathrm{L} \mathrm{NaNO}_{2}+5.0 \mathrm{~g} / \mathrm{L} \mathrm{CH}_{3} \mathrm{COONH}_{4}$ oxidizing solution were injected into the bath ${ }^{9)}$, as shown in Figure 1. The injection rate was $5 \mathrm{~cm}^{3} /$ min for both solutions. The mixed solution was homogenized using a temperature-controlled hot plate stirrer. We found that the plated film grew on the entire surface of the pellet at reaction temperatures of 25,50 , and $80^{\circ} \mathrm{C}$, while the $\mathrm{pH}$ of the oxidizing solution was controlled between 6.5 and 10 using $\mathrm{NH}_{3}$.

We evaluated the plated film by X-ray diffraction (XRD) measurements. Figure 2 shows an example of the obtained XRD curves. The measurement was carried out using a double-crystal X-ray diffractometer with a $\mathrm{Cu} \mathrm{K} \alpha \mathrm{X}$-ray source. The diffraction peaks were assigned as indexed in the figure, suggesting that ferrite was contained in the 
plated material. The full width at half maximum (FWHM) of the diffraction peak is a good index of the residual stress in a polycrystal. Figure 3 shows the relationship between the $\mathrm{pH}$ of the oxidizing solution and the FWHM of the ferrite (222) XRD peak. The figure indicates that the $\mathrm{pH}$ of the oxidizing solution did not affect the residual stress considerably. This characteristic is different in the case of nickel plating, where a clear correlation between the growth rate and the FWHM of the XRD peak exists ${ }^{7)}$. The insets show the appearances of the plated pellets. All the samples, except for the blackish samples formed at pHs 6.5 and 7, were brown, and there were no remarkable differences between them.

By optical microscope observation, we investigated the uniformity of the plated film thickness and the adhesion to the resin mold. Figure 4 shows a cross-sectional image of the plated film. For the observation, the samples were held using the adhesive resin. The photopolymerized resin had many pits $2-3 \mu \mathrm{m}$ in depth at the surface, and the pits were completely filled with the plated ferrite. The result indicates that this process is useful for the fabrication of 2 to $3 \mu \mathrm{m}$ structures. The resolution is of submicron order. We studied the growth rate in relation to the position of the plated film and the $\mathrm{pH}$, as shown in Figure 5. We can see that the growth rates were very different at the four faces of the mold. The dashed line in the figure indicates the mean value. The mean growth rate of the ferrite film was highest at $\mathrm{pH} 7$, but decreased at higher $\mathrm{pH}$ values. The amount of deposit increased and the deposit collected at the bottom of the bath at higher $\mathrm{pH}$ values. We consider that the reaction was so fast that much of the generated ferrite did not grow well as a film in that $\mathrm{pH}$ range. Generally, the higher the growth rate of the plated film, the more impurities and dislocations are introduced. The more impurities and dislocations are introduced, the wider the FWHM of XRD peak. 
However, this tendency did not manifest clearly in our experiments using ferrite.

The X-ray fluorescence was measured to evaluate the components of the plated film. As seen in Table I, the $\mathrm{pH}$ did not strongly affect the components. Considering the ratio of the atomic percent of $\mathrm{Co}_{\mathrm{x}} \mathrm{Fe}_{3-\mathrm{x}}$ and $\mathrm{O}_{4}$ in cobalt ferrite $\left(\mathrm{Co}_{\mathrm{x}} \mathrm{Fe}_{3-\mathrm{x}} \mathrm{O}_{4}\right)$, we can consider that $\mathrm{pH} 8$ is the best condition for cobalt ferrite formation. On the other hand, the temperature affected the components markedly. Oxygen became superabundant at low temperature and obstructed the ferrite formation. The tendency of ferrite formation roughly corresponded with the reduction in the FWHM.

\section{Copper Electroless Plating}

We also investigated the electroless plating of copper onto the photopolymer surface. The copper plating procedure is almost the same as that using nickel ${ }^{7)}$. After washing and catalysis, the sample was immersed in the plating solution. The composition of the catalytic solution was $0.8 \mathrm{~g} / \mathrm{L} \mathrm{PdCl} 2+15 \mathrm{~g} / \mathrm{L} \mathrm{SnCl}_{2}+150 \mathrm{~mL} / \mathrm{L}$ $\mathrm{HCl}$. The composition of the plating solution was $10 \mathrm{~g} / \mathrm{L} \mathrm{CuSO} \cdot 5 \mathrm{H}_{2} \mathrm{O}+20 \mathrm{~g} / \mathrm{L} \mathrm{CH}_{2} \mathrm{O}$ $+40 \mathrm{~g} / \mathrm{L} \mathrm{C}_{4} \mathrm{H}_{4} \mathrm{KNaO}_{6} \cdot 4 \mathrm{H}_{2} \mathrm{O}$ (Rochelle salt) $+15 \mathrm{~g} / \mathrm{L} \mathrm{H}_{2} \mathrm{NCH}_{2} \mathrm{COOH}$. The plating temperatures were 15,25 , and $40^{\circ} \mathrm{C}$, and the $\mathrm{pH}$ was controlled between 11.5 and 13 using $\mathrm{NaOH}$.

We measured the XRD to evaluate the characteristics of the plated film. The observed multiple peaks suggest that the plated film was composed of copper. Figure 6 shows the FWHM of the $\mathrm{Cu}(111)$ XRD peak. The figure clearly indicates that the FWHM increased as the $\mathrm{pH}$ of the plating solution was increased. The insets show the appearances of the plated samples under each condition. The metallic luster of the surface 
changed markedly. We can see in this figure that the crystal distortion increased with increasing $\mathrm{pH}$, but that the metallic luster decreased.

We observed the plated film using an optical microscope. The high-resolution image in Figure 7 indicates that the small surface pits were completely filled by the plated material. Through microscope observation, we evaluated the growth rate of the plated film, as shown in Figure 8. Although the growth rate differed according to the face of the pellet, there was a clear dependence on the $\mathrm{pH}$. The dashed line indicates the mean value. The growth rate of the plated film increased as the $\mathrm{pH}$ was increased. Considering that a high growth rate causes a large residual distortion in the crystal, we can understand Figure 6 by examining Figure 8 . On the other hand, although the growth rate of the plated film increased as the temperature was increased, the FWHM was smaller at $40^{\circ} \mathrm{C}$ than at $25^{\circ} \mathrm{C}$ (see Fig. 6). We attribute this to the surface diffusion occurring during plating. Surface diffusion is enhanced at high temperature, preventing the introduction of impurities and dislocations. We consider that the crystal distortion was determined by the growth speed, which competed with the surface diffusion speed during the copper film formation.

The X-ray fluorescence was measured to evaluate the components of the plated film (Table II). We found that the purity of the plated film was clearly related to the surface metallic luster. The copper purity increased as the $\mathrm{pH}$ was increased, reaching its highest value at $25^{\circ} \mathrm{C}$ at these temperatures, and was the lowest at $40^{\circ} \mathrm{C}$ where oxidation proceeded with most difficulty. The dependence of the copper purity on the $\mathrm{pH}$ and temperature closely matched the FWHM. In other words, many crystal distortions remained in the pure copper. 


\section{Fabrication Using a Resin Mold}

We fabricated a screw using a resin mold. Figure 9 shows the original screw, the mold and the fabricated copper structure. The resin mold was formed by replicating a screw made of stainless steel. In the formation process, we inserted a screw in raw resin and took out the screw after UV-light irradiation. Cracks and deformations were generated in the mold during the extraction of the original screw. The warped shape is difficult to discern in Figure 9(b), but is slightly more visible in Figure 9(c). Figure 9(d) shows the replica of the screw made of copper. The replica was extracted by cutting the plated film at one side of the mold and immersing it in acetone for two days. The copper screw came off from the mold because the resin expanded in the acetone but the copper did not change in size. On the other hand, the ferrite screw did not come out of the mold well. The fabricated copper screw had a glossy surface. The shape of the replica was distorted, but it traced the shape of the mold accurately. No shape distortion occurs when the resin mold is fabricated by optical stereolithography.

We next confirmed the structural reproducibility of the details. Figures 10(a) and 10(b) show high-resolution optical microscope images of the thread of the original screw and that of the copper replica, respectively. The thickness of the original thread was approximately 37 to $39 \mu \mathrm{m}$, and that of the copper replica was the same or slightly larger in some places. The discrepancy between the thicknesses is attributable to the deformation of the resin mold. We consider that the result demonstrates a transcriptional accuracy of micrometer scale. 


\section{Summary}

The electroless plating of photopolymerized resin with ferrite and copper was investigated with the aim of microfabricating three-dimensional structures. Evaluation by XRD and X-ray fluorescence measurements clarified that the resin mold was successfully plated with the materials by controlling the temperature and $\mathrm{pH}$ of the solution. High-resolution optical microscope observation demonstrated the applicability of this microfabrication process to the fabrication of micron-scale structures. We performed three-dimensional structure transcription, and succeeded in the fabrication of a screw by copper plating. We found that the $40-\mu \mathrm{m}$-thick thread was formed accurately. The ability to form three-dimensional microstructures made of various types of material will open up various application fields of micromachining, owing to the material properties of such microstructures, including mechanical strength, magnetism and high conductivity.

\section{References}

1) S. Maruo and S. Kawata: J. Microelectromech. Syst. 7 (1998) 411.

2) L. Wu, Y. Zhong, C. T. Chan, K. S. Wong, and G. P. Wang: Appl. Phys. Lett. 86 (2005) 241102.

3) S. Kawata, H.-B. Sun, T. Tanaka, and K. Takada: Nature 412 (2001) 697.

4) K. Nishimura, H. Uchida, M. Inoue, M. Sendoh, K. Ishiyama, and K. I. Arai: J. Appl. Phys. 93 (2003) 6712.

5) F. Formanek, N. Takeyasu, T. Tanaka, K. Chiyoda, A. Ishikawa, and S. Kawata: Appl. Phys. Lett. 88 (2006) 083110. 
6) T. Tanaka, A. Ishikawa, and S. Kawata: Appl. Phys. Lett. 88 (2006) 081107.

7) K. Mukai, S. Maruo, and T. Yoshimura: Jpn. J. Appl. Phys. 46 (2007) 2761.

8) K. Mukai, T Yoshimura, S. Kitayama, and S. Maruo: J. of Photopolym. Sci. Technol. 20 (2007) 285.

9) M. Abe, Y. Tamaura, and T. Itoh: Thin Solid Films, 216 (1992) 155.

\section{Figure Captions}

Figure 1 Schematic of experimental equipment for electroless plating using ferrite.

The inset shows the sample before plating.

Figure 2 XRD curve of ferrite plated onto resin mold in an oxidizing solution of $80^{\circ} \mathrm{C}$ at $\mathrm{pH} 8$.

Figure 3 FWHM of ferrite (222) peak as a function of $\mathrm{pH}$ of oxidizing solution. The insets show the appearances of the plated samples.

Figure 4 Cross-sectional optical microscope image of ferrite film.

Figure 5 Growth rate of ferrite-plated film as a function of $\mathrm{pH}$ of oxidizing solution.

Figure 6 FWHM of $\mathrm{Cu}(111)$ peak as a function of $\mathrm{pH}$ of plating solution. The insets show the appearances of the plated samples. 
Figure 7 Cross-sectional optical microscope image of copper film.

Figure 8 Growth rate of copper-plated film as a function of $\mathrm{pH}$ of plating solution.

Figure 9 Images of a sample during molding process: (a) original screw made of stainless steel, (b) resin mold of screw shape, (c) resin mold plated with copper, and (d) extracted screw made of copper.

Figure 10 Optical microscope images of thread of (a) original screw and (b) that of copper replica.

Table I Component elements measured by X-ray fluorescence, and FWHM of XRD (222) peak of ferrite film: $\mathrm{pH}$ and temperature dependences.

Table II Component elements measured by X-ray fluorescence, and FWHM of XRD (111) peak of copper film: $\mathrm{pH}$ and temperature dependences. 
Table I Component elements measured by X-ray fluorescence, and FWHM of XRD (222) peak of ferrite film: $\mathrm{pH}$ and temperature dependences.

\begin{tabular}{cccccc}
\hline at.\% & $\mathrm{pH} \mathrm{6.5}$ & $\mathrm{pH} \mathrm{7}$ & $\mathrm{pH} \mathrm{8}$ & $\mathrm{pH} \mathrm{9}$ & $\mathrm{pH} \mathrm{10}$ \\
\hline $\mathrm{Fe}$ & 52.3 & 44.7 & 39.9 & 50.3 & 31.5 \\
$\mathrm{Co}$ & 3.04 & 3.28 & 2.64 & 2.87 & 3.8 \\
$\mathrm{O}$ & 41 & 48.9 & 51.4 & 42.7 & 58.4 \\
$\mathrm{Cl}$ & 3.02 & 2.45 & 4.35 & 3.52 & 5.27 \\
\hline $\mathrm{FWHM}$ & 0.25 & 0.2 & 0.2 & 0.2 & 0.25 \\
\hline \multicolumn{6}{c}{} \\
\hline at.\% & $25^{\circ} \mathrm{C}$ & $50^{\circ} \mathrm{C}$ & $80^{\circ} \mathrm{C}$ \\
\hline $\mathrm{Fe}$ & 13.3 & 21.9 & 50.3 \\
$\mathrm{Co}$ & 0.76 & 1.31 & & 2.87 \\
$\mathrm{O}$ & 77.6 & 70.3 & & 42.7 \\
$\mathrm{Cl}$ & 7.66 & 5.34 & 3.52 \\
\hline $\mathrm{FWHM}$ & 0.25 & 0.25 & 0.2 \\
\hline
\end{tabular}


Table II Component elements measured by X-ray

fluorescence, and FWHM of XRD (111) peak of copper film: $\mathrm{pH}$ and temperature dependences.

\begin{tabular}{cccc}
\hline at.\% & $\mathrm{pH} \mathrm{11.5} \mathrm{12}$ & $\mathrm{pH} \mathrm{12} \mathrm{12.5}$ & $\mathrm{pH} \mathrm{12.5} \mathrm{13}$ \\
\hline $\mathrm{Cu}$ & 40.4 & 45.3 & 58.4 \\
$\mathrm{O}$ & 32.1 & 31.9 & 21.2 \\
$\mathrm{Na}$ & 14.6 & 10.2 & 12.8 \\
$\mathrm{Cl}$ & 8.27 & 6.13 & 3.9 \\
\hline $\mathrm{FWHM}$ & 0.25 & 0.3 & 0.45 \\
\hline
\end{tabular}

\begin{tabular}{cccc}
\hline at.\% & $15^{\circ} \mathrm{C}$ & $25^{\circ} \mathrm{C}$ & $40^{\circ} \mathrm{C}$ \\
\hline $\mathrm{Cu}$ & 53.6 & 58.4 & 36.3 \\
$\mathrm{O}$ & 22.2 & 21.2 & 47.5 \\
$\mathrm{Na}$ & 4.38 & 12.8 & 10.5 \\
$\mathrm{Cl}$ & 16.5 & 3.9 & 1.9 \\
\hline $\mathrm{FWHM}$ & 0.4 & 0.45 & 0.25 \\
\hline
\end{tabular}




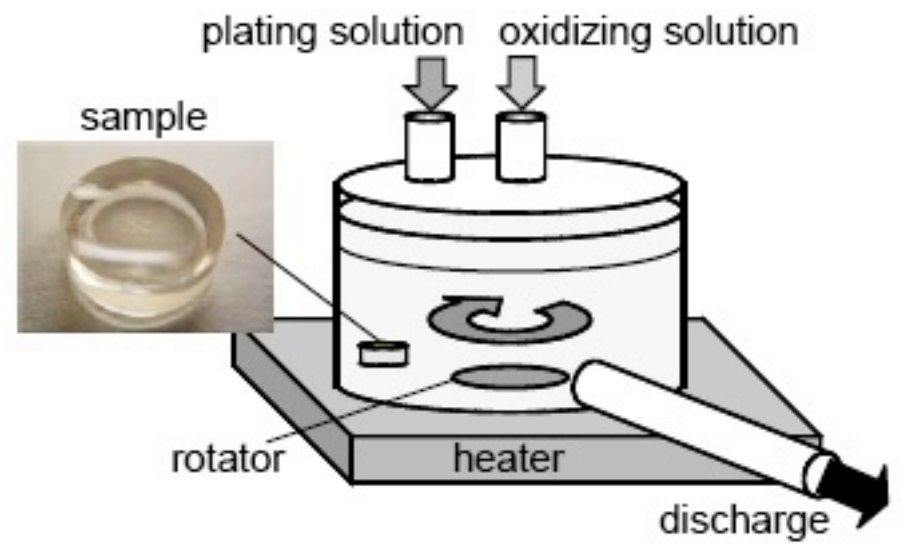

Figure 1 


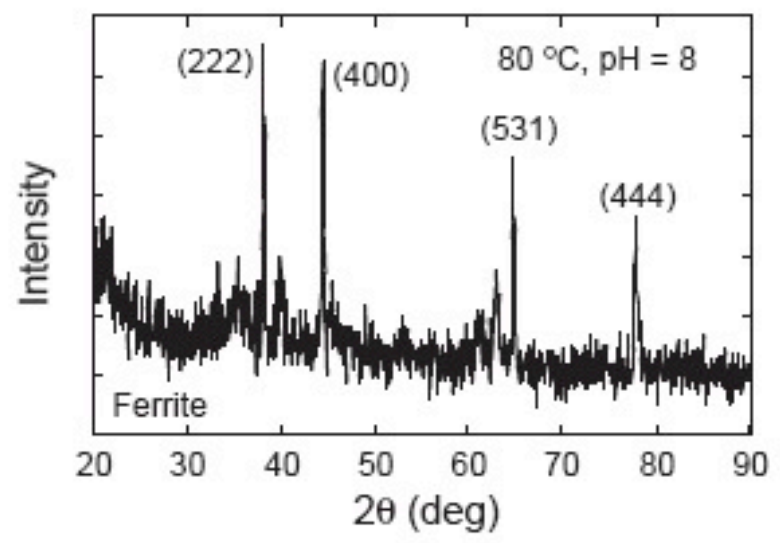

Figure 2 


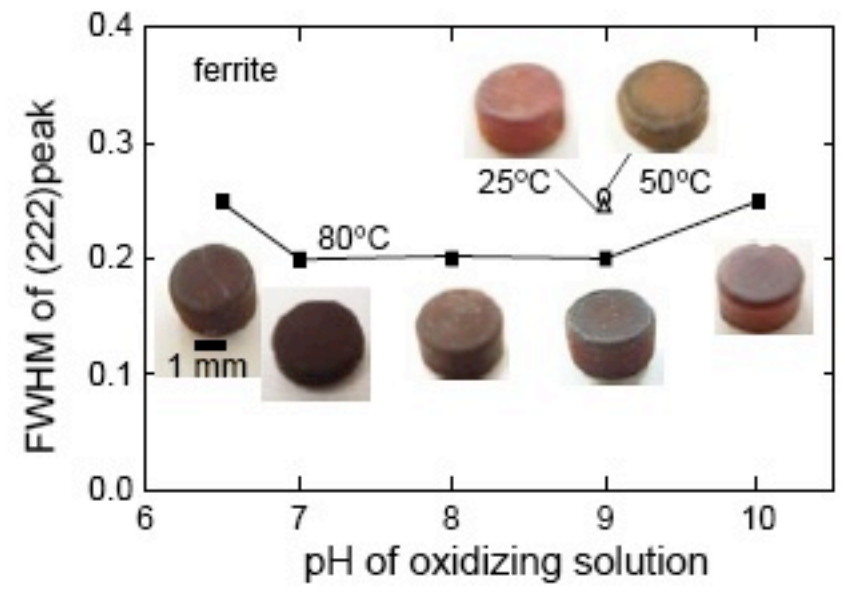

Figure 3 


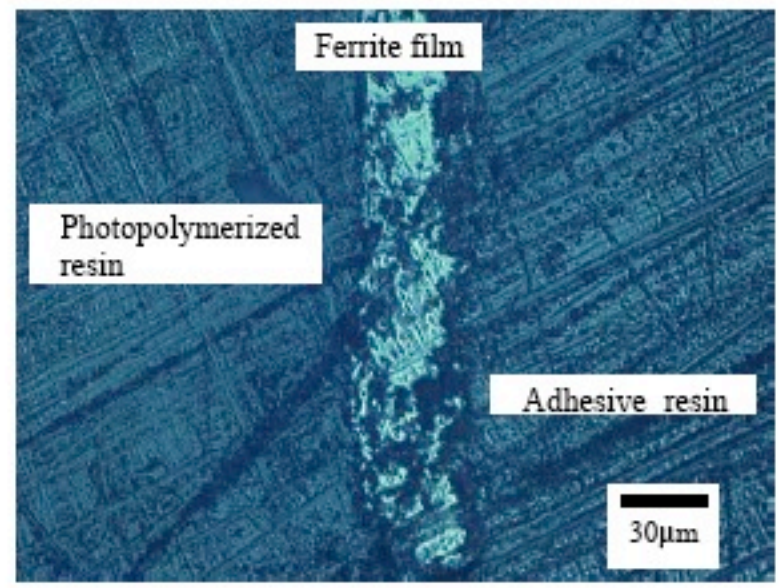

Figure 4 


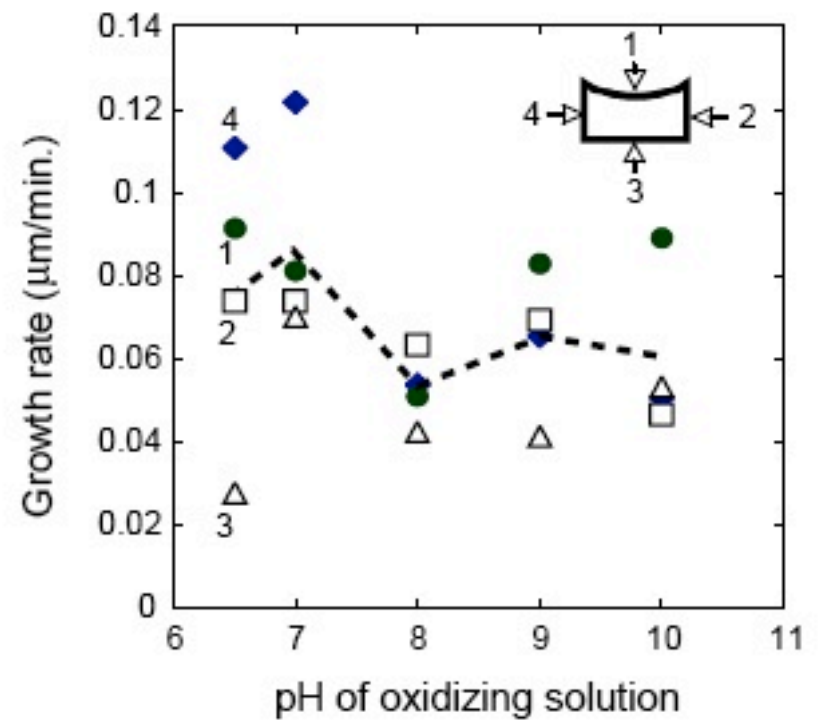

Figure 5 


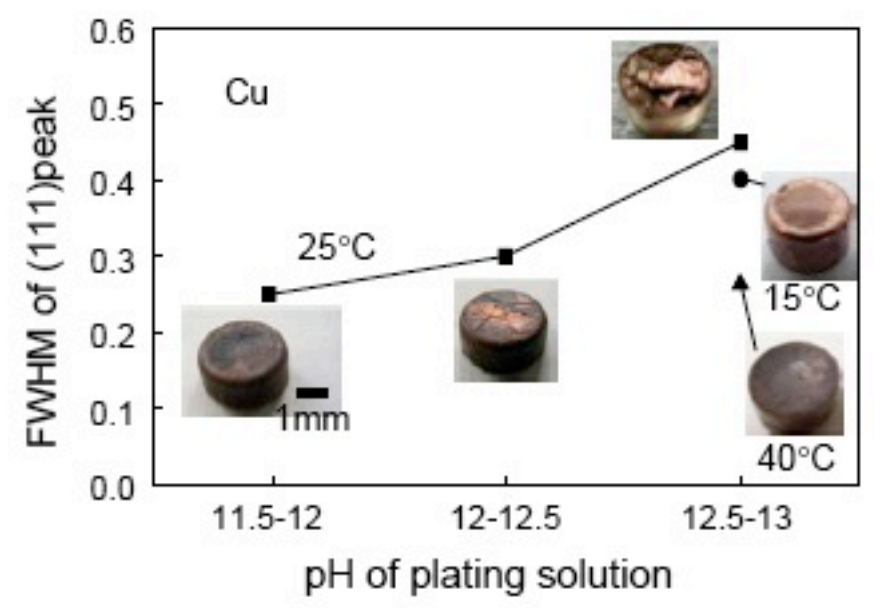

Figure 6 


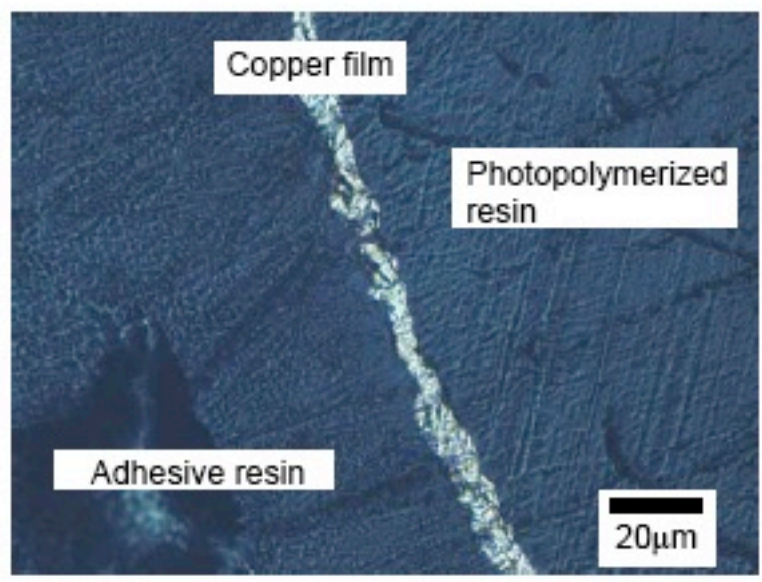

Figure 7 


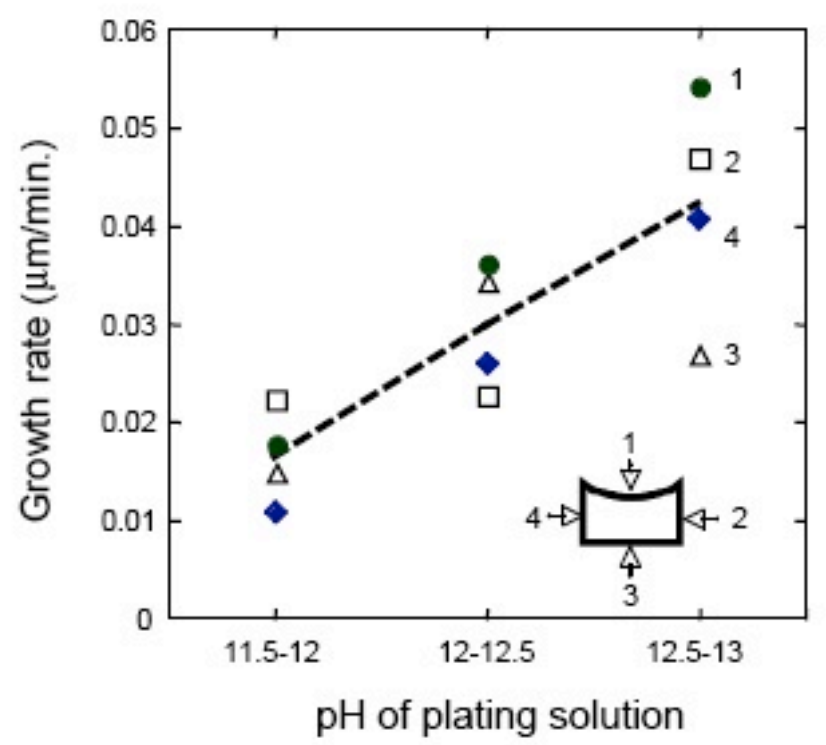

Figure 8 


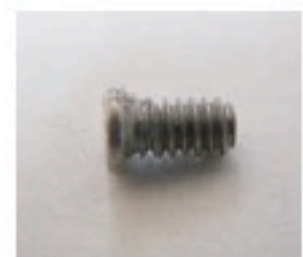

(a)

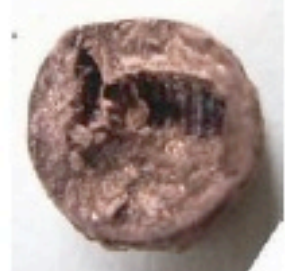

(c)

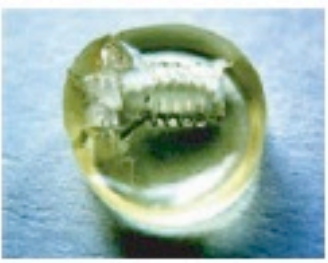

(b)

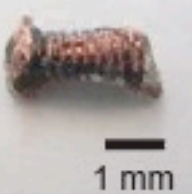

(d)

Figure 9 


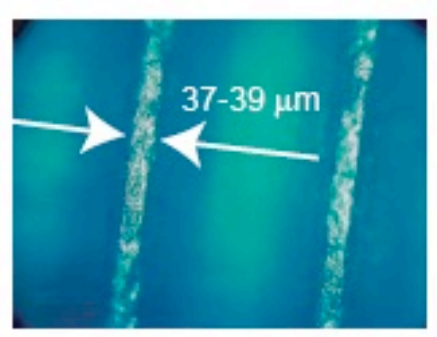

(a)

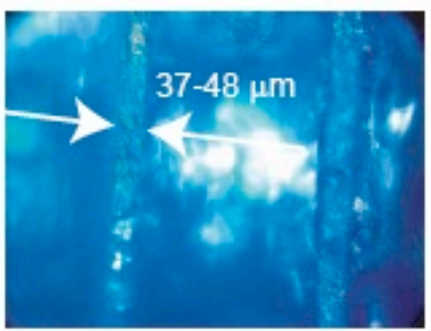

(b)

Figure 10 